University of Wollongong

Research Online

Faculty of Engineering and Information

Faculty of Engineering and Information

Sciences - Papers: Part A

Sciences

$1-1-2007$

Feature subset selection for multi-class SVM based image classification

Lei Wang

Australian National University, leiw@uow.edu.au

Follow this and additional works at: https://ro.uow.edu.au/eispapers

Part of the Engineering Commons, and the Science and Technology Studies Commons

Research Online is the open access institutional repository for the University of Wollongong. For further information contact the UOW Library: research-pubs@uow.edu.au 


\title{
Feature subset selection for multi-class SVM based image classification
}

\author{
Abstract \\ Multi-class image classification can benefit much from feature subset selection. This paper extends an \\ error bound of binary SVMs to a feature subset selection criterion for the multi-class SVMs. By minimizing \\ this criterion, the scale factors assigned to each feature in a kernel function are optimized to identify the \\ important features. This minimization problem can be efficiently solved by gradient-based search \\ techniques, even if hundreds of features are involved. Also, considering that image classification is often \\ a small sample problem, the regularization issue is investigated for this criterion, showing its robustness \\ in this situation. Experimental study on multiple benchmark image data sets demonstrates the \\ effectiveness of the proposed approach.

\section{Keywords} \\ svm, class, multi, selection, subset, image, feature, classification \\ Disciplines \\ Engineering | Science and Technology Studies

\section{Publication Details} \\ Wang, L. (2007). Feature subset selection for multi-class SVM based image classification. Lecture Notes \\ in Computer Science, 4844 145-154.
}




\title{
Feature Subset Selection for Multi-class SVM Based Image Classification
}

\author{
Lei Wang \\ Research School of Information Sciences and Engineering \\ The Australia National University \\ Australia, ACT, 0200
}

\begin{abstract}
Multi-class image classification can benefit much from feature subset selection. This paper extends an error bound of binary SVMs to a feature subset selection criterion for the multi-class SVMs. By minimizing this criterion, the scale factors assigned to each feature in a kernel function are optimized to identify the important features. This minimization problem can be efficiently solved by gradient-based search techniques, even if hundreds of features are involved. Also, considering that image classification is often a small sample problem, the regularization issue is investigated for this criterion, showing its robustness in this situation. Experimental study on multiple benchmark image data sets demonstrates the effectiveness of the proposed approach.
\end{abstract}

\section{Introduction}

Multi-class image classification often involves (i) high-dimensional feature vectors. An image is often reshaped as a long vector, leading to hundreds of dimensions; (ii) redundant information. Only part of an image, for instance, the foreground, is really useful for classification. In the past few years, multi-class Support Vector Machines (SVMs) have been successfully applied to image classification [12. However, the feature subset selection issue, that is, identifying $p$ important features from the original $d$ ones has not been paid enough attention 1 Classical feature subset selection techniques may be used to find the important dimensions. For instance, in [3], a subset of 15 features is selected by using the wrapper approach from 29 features. However, this approach often has heavy computational load and cannot deal with image classification generally having hundreds of features. Also, the selected features may not fit the SVM classifier well when the selection criteria have no connection with the SVMs.

Recent advance in the binary SVMs provides a ray of hope. In [4], a leave-oneout error bound of the binary SVMs (the radius-margin bound) is minimized to optimize the kernel parameters. The minimization is solved by gradient-based search techniques which can handle a large number of free kernel parameters.

\footnotetext{
${ }^{1}$ Please note that this is different from the problem of finding the $p$ optimal combination of the original $d$ features, for instance, in the way of PCA or LDA. In feature subset selection, the features are kept individual and the feature dependence is left to the classifier, for instance, the SVMs, which handles it automatically.
} 
Also, it is found that the optimized kernel parameters can reflect the importance of features for classification. These properties are just what we are seeking for. Unfortunately, this error bound cannot be straightforwardly applied to the multiclass scenario because it is rooted in the theoretical result of binary SVMs, for instance, the VC-Dimension theory upon which it is developed. A possible way may be to apply the radius-margin bound to all of the binary SVMs obtained in a decomposition of the multi-class SVMs. However, by doing so, the selected features will be good at discriminating a certain pair of classes only. Also, how to integrate these selection results is another issue. Hence, this paper seeks a one-shot feature selection from the perspective of multi-class classification.

To realize this, this paper extends the radius-margin bound to a feature subset selection criterion for the multi-class SVMs. Firstly, the relationship between the binary radius-margin bound and the classical class separability concept is discussed. Enlightened by it, this work redefines the radius and margin to accommodate multiple classes, forming a new criterion. Its derivative with respect to the kernel parameters can also be analytically calculated, making the gradient-based search techniques still applicable. Minimization of this criterion can efficiently optimize hundreds of kernel parameters simultaneously. These optimized parameters are then used to identify the important features. This approach is quite meaningful for practical image classification because it facilitates the reduction of system complexity and the feature discovery from the perspective of multiclass classification. Experimental result on benchmark data sets demonstrates the effectiveness of this approach.

\section{Background}

Let $\mathcal{D}$ denote a set of $m$ training samples and $\mathcal{D}=(\mathbf{x}, y) \in\left(\mathbb{R}^{d} \times \mathcal{Y}\right)^{m}$, where $\mathbb{R}^{d}$ denotes a $d$-dimensional input space, $\mathcal{Y}$ denotes the label set of $\mathbf{x}$, and the size of $\mathcal{Y}$ is the number of classes, c. A kernel is defined as $k_{\boldsymbol{\theta}}\left(\mathbf{x}_{i}, \mathbf{x}_{j}\right)=\left\langle\phi\left(\mathbf{x}_{i}\right), \phi\left(\mathbf{x}_{j}\right)\right\rangle$, where $\phi(\cdot)$ is a possibly nonlinear mapping from $\mathbb{R}^{d}$ to a feature space, $\mathcal{F}$, and $\boldsymbol{\theta}$ is the kernel parameter set. Due to the limit of space, the introduction of multi-class SVMs is omitted and this information can be found from [12].

The radius-margin bound. It is an upper bound of the test error estimated via a leave-one-out cross-validation procedure. Let $\mathcal{L}_{m}=\mathcal{L}\left(\left(\mathbf{x}_{1}, y_{1}\right), \cdots,\left(\mathbf{x}_{m}, y_{m}\right)\right)$ be the number of errors in this procedure. It is shown in [4] that

$$
\mathcal{L}_{m} \leq \frac{4 R^{2}}{\gamma^{2}}=4 R^{2}\|\mathbf{w}\|^{2}
$$

where $R$ is the radius of the smallest sphere enclosing all the $m$ training samples in $\mathcal{F}, \gamma$ the margin, w the normal vector of the optimal separating hyperplane, and $\gamma^{-1}=\|\mathbf{w}\|$. The $R^{2}$ is obtained by solving

$$
\begin{aligned}
& R^{2}=\max _{\boldsymbol{\beta} \in \mathbb{R}^{m}}\left[\sum_{i=1}^{m} \beta_{i} k_{\boldsymbol{\theta}}\left(\mathbf{x}_{i}, \mathbf{x}_{i}\right)-\sum_{i, j=1}^{m} \beta_{i} \beta_{j} k_{\boldsymbol{\theta}}\left(\mathbf{x}_{i}, \mathbf{x}_{j}\right)\right] \\
& \text { subject to }: \sum_{i=1}^{m} \beta_{i}=1 ; \beta_{i} \geq 0(i=1,2, \cdots, m)
\end{aligned}
$$


where $\beta_{i}$ is the $i$-th Lagrange multiplier. The $\|\mathbf{w}\|^{2}$ is obtained by solving

$$
\begin{aligned}
& \frac{1}{2}\|\mathbf{w}\|^{2}=\max _{\boldsymbol{\alpha} \in \mathbb{R}^{m}}\left[\sum_{i=1}^{m} \alpha_{i}-\frac{1}{2} \sum_{i, j=1}^{m} \alpha_{i} \alpha_{j} y_{i} y_{j} k_{\boldsymbol{\theta}}\left(\mathbf{x}_{i}, \mathbf{x}_{j}\right)\right] \\
& \text { subject to }: \sum_{i=1}^{m} \alpha_{i} y_{i}=0 ; \alpha_{i} \geq 0(i=1,2, \cdots, m)
\end{aligned}
$$

where $\alpha_{i}$ is the $i$-th Lagrange multiplier. The derivative of $R^{2}$ w.r.t. the $t$-th kernel parameter, $\theta_{t}$, is shown in [4] as

$$
\frac{\partial R^{2}}{\partial \theta_{t}}=\sum_{i=1}^{m} \beta_{i}^{0} \frac{\partial k_{\boldsymbol{\theta}}\left(\mathbf{x}_{i}, \mathbf{x}_{i}\right)}{\partial \theta_{t}}-\sum_{i, j=1}^{m} \beta_{i}^{0} \beta_{j}^{0} \frac{\partial k_{\boldsymbol{\theta}}\left(\mathbf{x}_{i}, \mathbf{x}_{j}\right)}{\partial \theta_{t}}
$$

where $\beta_{i}^{0}$ is the solution of Eq. (2). Similarly, the derivative of $\|\mathbf{w}\|^{2}$ w.r.t. $\theta_{t}$ is

$$
\frac{\partial\|\mathbf{w}\|^{2}}{\partial \theta_{t}}=(-1) \cdot \sum_{i, j=1}^{m} \alpha_{i}^{0} \alpha_{j}^{0} y_{i} y_{j} \frac{\partial k_{\boldsymbol{\theta}}\left(\mathbf{x}_{i}, \mathbf{x}_{j}\right)}{\partial \theta_{t}}
$$

where $y_{i} \in\{+1,-1\}$ is the label of $\mathbf{x}_{i}$. Thus, the radius-margin bound can be efficiently minimized by using the gradient search methods. As seen, this bound is rooted in binary SVMs and cannot be directly applied to the multi-class case. An extension of this bound to a feature selection criterion for multi-class SVMs is proposed below.

\section{Proposed Approach}

Class separability is a concept widely used in pattern recognition. The scatter matrix based measure is often favored thanks to its simplicity. The Betweenclass scatter matrix $\left(\mathbf{S}_{B}\right)$ and Total scatter matrix $\left(\mathbf{S}_{T}\right)$ are defined as $\mathbf{S}_{B}=$ $\sum_{i=1}^{c} n_{i}\left(\mathbf{m}_{i}-\mathbf{m}\right)\left(\mathbf{m}_{i}-\mathbf{m}\right)^{\top}$ and $\mathbf{S}_{T}=\sum_{i=1}^{c}\left[\sum_{j=1}^{n_{i}}\left(\mathbf{x}_{i j}-\mathbf{m}\right)\left(\mathbf{x}_{i j}-\mathbf{m}\right)^{\top}\right]$, where $n_{i}$ is the size of class $i, \mathbf{x}_{i j}$ the $j$-th sample of class $i, \mathbf{m}_{i}$ the mean of class $i$, and $\mathbf{m}$ the mean of all $c$ classes. The following derives the class separability in the feature space, $\mathcal{F}$. Let $\mathcal{D}_{i}$ be the training samples from class $i$ and $\mathcal{D}$ be the set of all training samples. The $\mathbf{m}_{i}^{\phi}$ and $\mathbf{m}^{\phi}$ are the mean vectors of $\mathcal{D}_{i}$ and $\mathcal{D}$ in $\mathcal{F}$, respectively. $\mathbf{K}_{\mathcal{A}, \mathcal{B}}$ is the kernel matrix where $\left\{\mathbf{K}_{\mathcal{A}, \mathcal{B}}\right\}_{i j}=k_{\boldsymbol{\theta}}\left(\mathbf{x}_{i}, \mathbf{x}_{j}\right)$ with the constraints of $\mathbf{x}_{i} \in \mathcal{A}$ and $\mathbf{x}_{j} \in \mathcal{B}$. The traces of $\mathbf{S}_{B}^{\phi}$ and $\mathbf{S}_{T}^{\phi}$ can be expressed as

$$
\operatorname{tr}\left(\mathbf{S}_{B}^{\phi}\right)=\sum_{i=1}^{c} \frac{\mathbf{1}^{\top} \mathbf{K}_{\mathcal{D}_{i}, \mathcal{D}_{i}} \mathbf{1}}{n_{i}}-\frac{\mathbf{1}^{\top} \mathbf{K}_{\mathcal{D}, \mathcal{D}} \mathbf{1}}{n} ; \operatorname{tr}\left(\mathbf{S}_{T}^{\phi}\right)=\operatorname{tr}\left(\mathbf{K}_{\mathcal{D}, \mathcal{D}}\right)-\frac{\mathbf{1}^{\top} \mathbf{K}_{\mathcal{D}, \mathcal{D}} \mathbf{1}}{n}
$$

where $n=\sum_{i=1}^{c} n_{i}$ and $\mathbf{1}$ is a column vector whose components are all 1 . Based on these, the class separability in $\mathcal{F}$ can be written as $\mathcal{C}=\frac{\operatorname{tr}\left(\mathbf{S}_{B}^{\phi}\right)}{\operatorname{tr}\left(\mathbf{S}_{T}^{\phi}\right)}$. In the case of two classes, it can be shown that

$$
\operatorname{tr}\left(\mathbf{S}_{B}^{\phi}\right)=\left(\frac{n_{1} n_{2}}{n_{1}+n_{2}}\right)\left\|\mathbf{m}_{1}^{\phi}-\mathbf{m}_{2}^{\phi}\right\|^{2} ; \quad \operatorname{tr}\left(\mathbf{S}_{T}^{\phi}\right)=\sum_{i=1}^{2} \sum_{j=1}^{n_{i}}\left\|\phi\left(\mathbf{x}_{i j}\right)-\mathbf{m}^{\phi}\right\|^{2}
$$


and it can be proven (the proof is omitted) that

$$
\gamma^{2} \leq \frac{1}{4-\left(\frac{n_{1}+n_{2}}{n_{1} n_{2}}\right) \operatorname{tr}\left(\mathbf{S}_{B}^{\phi}\right)} ; \quad R^{2} \geq \frac{1}{\left(n_{1}+n_{2}\right)} \operatorname{tr}\left(\mathbf{S}_{T}^{\phi}\right)
$$

That is, $\operatorname{tr}\left(\mathbf{S}_{B}^{\phi}\right)$ measures the squared distance between the class means in $\mathcal{F} \cdot \operatorname{tr}\left(\mathbf{S}_{T}^{\phi}\right)$ measures the squared scattering radius of the training samples in $\mathcal{F}$ if divided by $\left(n_{1}+n_{2}\right)$. Conceptually, the $\left\|\mathbf{m}_{1}^{\phi}-\mathbf{m}_{2}^{\phi}\right\|^{2}$ and $\gamma^{2}$ reflect the similar property of data separability and $\operatorname{tr}\left(\mathbf{S}_{T}^{\phi}\right)$ positively correlates with $R^{2}$. Theoretically, $\gamma^{2}$ is upper bounded by a functional of $\operatorname{tr}\left(\mathbf{S}_{B}^{\phi}\right)$ and $R^{2}$ is lower bounded by $\operatorname{tr}\left(\mathbf{S}_{T}^{\phi}\right) /\left(n_{1}+n_{2}\right)$. When minimizing the radius-margin bound, $\gamma^{2}$ is maximized while $R^{2}$ is minimized. This requires $\operatorname{tr}\left(\mathbf{S}_{B}^{\phi}\right)$ to be maximized and $\operatorname{tr}\left(\mathbf{S}_{T}^{\phi}\right)$ to be minimized (although this does not necessarily have $\gamma^{2}$ maximized or $R^{2}$ minimized). Hence, minimizing the radius-margin bound can be viewed as approximately maximizing the class separability in $\mathcal{F}$. Part of the analysis can also be seen in 5 .

Enlightened by this analogy, this work extends the radius-margin bound to accommodate multiple classes. In the multi-class case, $\operatorname{tr}\left(\mathbf{S}_{T}^{\phi}\right) / n$ measures the squared scattering radius of all training samples in $\mathcal{F}$. Considering the analogy between $\operatorname{tr}\left(\mathbf{S}_{T}^{\phi}\right)$ and $R^{2}$, the $R^{2}$ is redefined as the squared radius of the sphere enclosing all the training samples in the $c$ classes,

$$
R_{c}^{2}=\min _{\left\|\phi\left(\mathbf{x}_{i}\right)-\hat{\mathbf{c}}\right\|^{2} \leq \hat{R}^{2}, \forall \mathbf{x}_{i} \in \mathcal{D}}\left(\hat{R}^{2}\right) .
$$

For $\operatorname{tr}\left(\mathbf{S}_{B}^{\phi}\right)$ in the multi-class case, it can be proven that

$$
\operatorname{tr}\left(\mathbf{S}_{B}^{\phi}\right)=\frac{\sum_{1 \leq i<j \leq c} n_{i} n_{j}\left\|\mathbf{m}_{i}^{\phi}-\mathbf{m}_{j}^{\phi}\right\|^{2}}{n} .
$$

Noting the analogy between $\left\|\mathbf{m}_{1}^{\phi}-\mathbf{m}_{2}^{\phi}\right\|^{2}$ and $\gamma^{2}$, the margin is redefined as

$$
\gamma_{c}^{2}=\sum_{1 \leq i<j \leq c} P_{i} P_{j} \gamma_{i j}^{2}=\sum_{1 \leq i<j \leq c} P_{i} P_{j}\left\|\mathbf{w}_{i j}\right\|^{-2}
$$

where $\gamma_{i j}$ is the margin between class $i$ and $j$, and $P_{i}=\frac{n_{i}}{n}$, which is the priori probability of the $i$-th class estimated from the training data. By doing so, a feature selection criterion for multi-class SVMs is obtained. The optimal kernel parameters is sought by minimizing this criterion,

$$
\boldsymbol{\theta}^{\star}=\arg \min _{\boldsymbol{\theta} \in \boldsymbol{\Theta}}\left(\frac{R_{c}^{2}}{\gamma_{c}^{2}}\right) \text {. }
$$

The derivation of this criterion w.r.t. the $t$-th kernel parameter, $\theta_{t}$, is

$$
\frac{\partial}{\partial \theta_{t}}\left(\frac{R_{c}^{2}}{\gamma_{c}^{2}}\right)=\frac{1}{\gamma_{c}^{4}}\left(\gamma_{c}^{2} \frac{\partial R_{c}^{2}}{\partial \theta_{t}}-R_{c}^{2} \frac{\partial \gamma_{c}^{2}}{\partial \theta_{t}}\right) .
$$


The calculation of $\frac{\partial R_{c}^{2}}{\partial \theta_{t}}$ and $\frac{\partial\left\|\mathbf{w}_{i j}\right\|^{2}}{\partial \theta_{t}}$ follows the Eq. (4) and (5). This criterion can still be minimized by using the gradient-based search techniques. Finally, please note that the obtained criterion is not necessarily an upper bound of the leave-one-out error of the multi-class SVMs. It is only a criterion reflecting the idea of the radius-margin bound and working for the multi-class case. An elegant extension of the radius-margin bound to multi-class SVMs is in [6].

Regularization issue. When optimizing a criterion with multiple parameters, regularization is often needed to avoid over-fitting the training samples, especially when the number of training samples is small. To check whether this is also needed for this new criterion, a regularized version is given as

$$
\mathcal{J}(\boldsymbol{\theta})=(1-\lambda) \frac{R_{c}^{2}}{\gamma_{c}^{2}}+\lambda\left\|\boldsymbol{\theta}-\boldsymbol{\theta}_{0}\right\|^{2}
$$

where $\lambda(0 \leq \lambda<1)$ is the regularization parameter which penalizes the deviation of $\boldsymbol{\theta}$ from a preset $\boldsymbol{\theta}_{0}$. Such a regularization imposes a Gaussian prior over the parameter $\boldsymbol{\theta}$ to be estimated. When using the ellipsoid Gaussian RBF kernel defined in Section $4, \boldsymbol{\theta}_{0}$ can be chosen by applying the constraint of $\theta_{1}=\theta_{2}=\cdots=\theta_{d}$ and solving

$$
\boldsymbol{\theta}_{0}^{\star}=\left.\arg \min _{\boldsymbol{\theta} \in \boldsymbol{\Theta}}\left(\frac{R_{c}^{2}}{\gamma_{c}^{2}}\right)\right|_{\theta_{1}=\theta_{2}=\cdots=\theta_{d}} .
$$

Since the number of free parameters reduces to one, over-fitting is less likely to occur. It is worth noting that the obtained $\boldsymbol{\theta}_{0}$ has been optimal for the minimization of $R_{c}^{2} / \gamma_{c}^{2}$ with a spherical Gaussian RBF kernel. It is a good starting point for the subsequent multi-parameter optimization.

\section{Experimental Result}

The elliptical Gaussian RBF kernel, $k(\mathbf{x}, \mathbf{y})=\exp \left(-\sum_{i=1}^{d} \frac{\left(x_{i}-y_{i}\right)^{2}}{2 \sigma_{i}^{2}}\right)$, is used. For binary SVMs, the optimized $\sigma_{i}$ can reflect the importance of the feature $i$ for classification [4. The larger the $\sigma_{i}$, the less important the feature $i$. Now, the multi-class case is studied with the proposed criterion. The BFGS Quasi-Newton optimization method is used. For the convenience of optimization, $g_{i}\left(g_{i}=1 / 2 \sigma_{i}^{2}\right)$ is optimized instead. A multi-class SVM classifier using the selected features is then trained and evaluated by using [7]. Following [4, the feature selection criteria of "Fisher score", "Pearson correlation coefficient" and "Kolmogorov-Smirnov test" are compared with the proposed criterion.

USPS for Optical digit recognition. This data set contains 7291 training images and 2007 test images from ten classes of digits of " 0 " to "9". Each $16 \times 16$ thumbnail image is reshaped to a 256-dimensional feature vector consisting of its gray values. The proposed feature selection criterion is minimized on the training set to optimize the $g_{i}$ for each dimension. The 256 features are then sorted in a descending order of the $g$ values, and the top $k$ features are used for 


\section{D112131815/677819}

(a) Optical character recognition

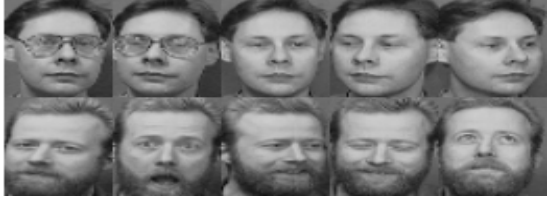

(b) Facial image classification

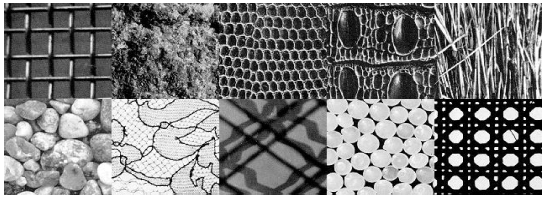

(c) Textured image classification

Fig. 1. Examples of image classification tasks

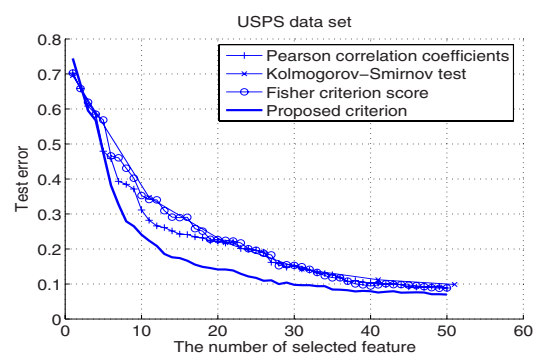

(a) Feature selection result

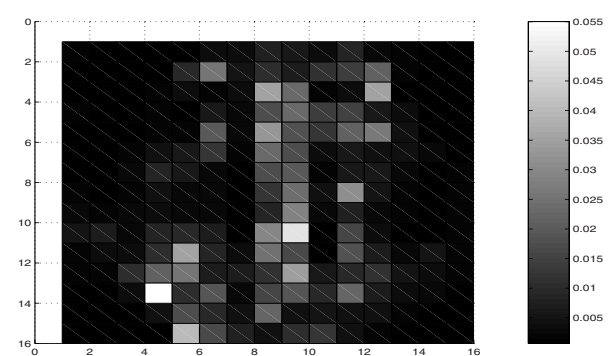

(b) Optimized value of $g=1 / 2 \sigma^{2}$

Fig. 2. Results for the USPS data set

classification. As shown in Figure 2(a), the test error for the proposed criterion decreases quickly with $k$. With only 50 features selected, it reaches $6.7 \%$ (The reported lowest error is $4.3 \%$ when all 256 features are used [8]). Compared with the other three selection criteria, this criterion gives the best feature selection performance. Now, the optimized $g_{1}, \cdots, g_{256}$ are reshaped back into a $16 \times 16$ matrix and shown in Figure 2(b). In this map, each block corresponds to one of the $256 \mathrm{~g}$ 's and its value is reflected by the gray level. As seen, the blocks with the larger $g$ values scatter over the central part of this map, whereas those with the lower values are mostly at the borders and corners. This suggests that the pixels at the borders and corners are less important for discrimination. This observation well matches the images in Figure 1(a) in that the digits are displayed at the central parts and surrounded by a uniform black background.

ORL for Facial image classification. This database contains 40 subjects and each of them has 10 gray-level facial images, as shown in Figure 1(b). Each image is resized to $16 \times 16$ and a 256 -dimensional feature vector is obtained. The 400 images are randomly split into 10 pairs of training/test subsets with the equal size of 200, forming ten 40-class classification problems. For each problem, the $g$ values are optimized on the training subset and the features are sorted accordingly. The feature selection result averaged over the 10 classification problems is reported in Figure 3(a). By using only the top 50 selected features, the 


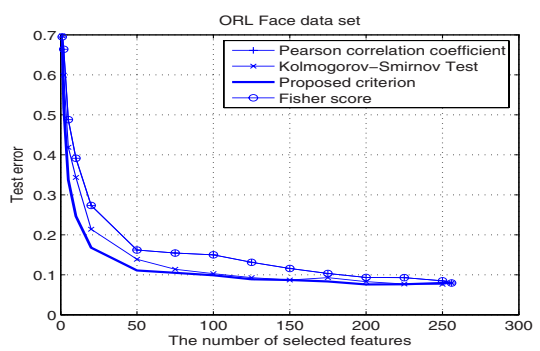

(a) Feature selection result

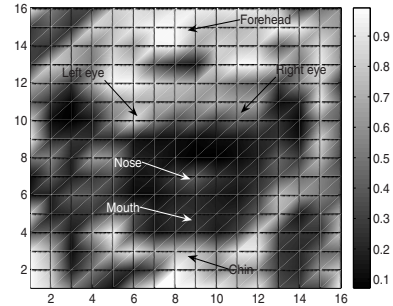

(b) Optimized value of $g=1 / 2 \sigma^{2}$

Fig. 3. Results for the ORL face data set

test error can be as low as $11.10 \pm 2.87 \%$, and the test error with all 256 features is $7.95 \pm 2.36 \%$. Again, the proposed criterion achieves the best selection performance, especially at the top 50. The optimized $g$ values are also reshaped back and plotted in Figure 3(a). A shading effect is used. As seen, the blocks with larger $g$ values (whiter) roughly present a human face, where the eyes, nose, mouth, forehead and chin are marked. This result is consistent with our daily experience that we recognize a person often by looking at the eyes while the cheek, shown as the darker areas, is less used. The subjects in this database often have different hairstyles at the forehead part, and this may explain why the forehead part is also shown as a whiter region.

Brodatz for Textured image classification. This database includes 112 different textured images, as shown in Figure 1(c). Top 10 images are used in this experiment. For each of them, sixteen $128 \times 128$ sub-images are cropped, without overlapping, from the original $512 \times 512$ image to form a texture class. By doing so, a ten-class textured image database is created, including 160 samples. Again, they are randomly split into 10 pairs of training (40 samples)/test (120 samples) subsets. By utilizing a bank of Gabor filters (6 orientations and 4 scales), the mean, variance, skewness and kurtosis of the values of the Gabor filtered images are extracted, leading to a $96(6 \times 4 \times 4)$ dimensional feature vector 9 . Using the proposed criterion, the $96 \mathrm{~g}$ values are optimized and the features are sorted. The test error with different number of selected features is plotted in Figure 4(a). This criterion and the "Kolmogorov-Smirnov test" show comparable performance, with the former slightly better at selecting the top 10 features. Using this criterion, the lowest test error of $1.42 \pm 1.36 \%$ is achieved at the top 50 selected features, whereas the error with all 96 features is $2.08 \pm 1.06 \%$. This indicates that half features are actually useless for discrimination. Again, the optimized $g$ values are shown in Figure 4(b). It is found that the features from the 25-th to 48-th (the features of "variance") and those from the 80-th to 96-th (the features of "kurtosis") dominate the features with larger $g$ values, suggesting that these two types of features are more useful for discriminating the textured images. 


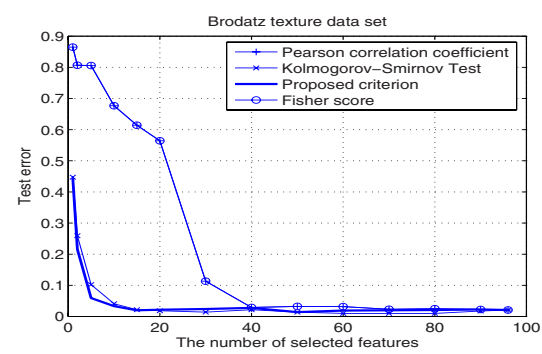

(a) Feature selection result

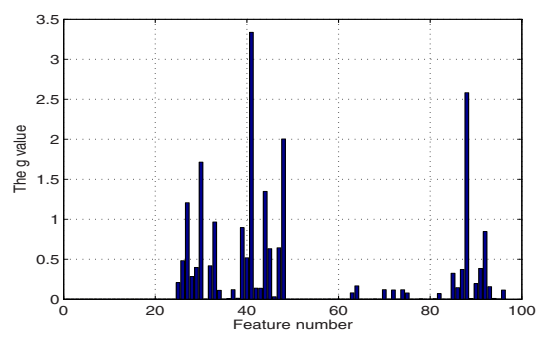

(b) Optimized value of $g=1 / 2 \sigma^{2}$

Fig. 4. Results for the Brodatz data set

Table 1. Feature selection performance with regularization (ORL data set)

\begin{tabular}{|l||c|c|c|c|c|c|}
\hline \multirow{2}{*}{\multicolumn{1}{|c||}{$\lambda$ value }} & \multicolumn{5}{c|}{ Average test error for different number of selected features (In \%) } \\
\cline { 2 - 7 } & 1 & 2 & 5 & 10 & 20 & 50 \\
\hline \hline 0 & $\mathbf{6 8 . 8 5} \pm \mathbf{2 . 7 3}$ & $\mathbf{5 1 . 8 0} \pm \mathbf{8 . 1 6}$ & $\mathbf{3 3 . 7 5} \pm \mathbf{3 . 2 8}$ & $\mathbf{2 4 . 6 5} \pm \mathbf{3 . 9 4}$ & $\mathbf{1 6 . 8 0} \pm \mathbf{3 . 2 8}$ & $\mathbf{1 1 . 1 0} \pm \mathbf{2 . 8 7}$ \\
\hline 0.1 & $70.10 \pm 4.01$ & $62.85 \pm 10.15$ & $40.70 \pm 4.74$ & $30.15 \pm 2.24$ & $19.60 \pm 3.85$ & $11.75 \pm 2.20$ \\
\hline 0.25 & $70.10 \pm 4.01$ & $62.05 \pm 11.10$ & $40.30 \pm 5.06$ & $28.80 \pm 1.46$ & $19.15 \pm 3.42$ & $12.20 \pm 2.38$ \\
\hline 0.5 & $73.50 \pm 5.25$ & $57.10 \pm 12.12$ & $36.05 \pm 5.23$ & $29.15 \pm 4.78$ & $20.20 \pm 3.12$ & $12.55 \pm 2.75$ \\
\hline 0.75 & $77.40 \pm 6.82$ & $53.85 \pm 9.92$ & $35.05 \pm 4.73$ & $28.80 \pm 4.19$ & $20.17 \pm 2.72$ & $12.95 \pm 3.07$ \\
\hline 0.9 & $74.45 \pm 6.09$ & $55.40 \pm 11.29$ & $35.60 \pm 4.40$ & $28.10 \pm 3.27$ & $21.25 \pm 2.44$ & $12.60 \pm 3.18$ \\
\hline 0.99 & $79.60 \pm 7.40$ & $64.15 \pm 8.67$ & $37.15 \pm 6.03$ & $28.25 \pm 3.43$ & $22.10 \pm 2.73$ & $12.85 \pm 3.35$ \\
\hline
\end{tabular}

Finally, the help of regularization is investigated for the proposed criterion on the ORL and Brodatz data sets. For the ORL, there are only 5 training samples in each class, whereas the number of features is 256 . For the Brodatz, the case is 4 versus 96 . Both have the small-sized training sets with respect to the number of features. In this experiment, $\boldsymbol{\theta}_{0}$ is obtained by solving Eq. (15). By gradually increasing the regularization parameter, $\lambda$, from 0 to 0.99 , the $g$ values are optimized and the features are selected. The average test error of a multi-class SVM classifier with the selected features is reported in Table 1. It is found that the lowest error (in bold) is consistently obtained with $\lambda=0$, that is, no regularization is imposed. Simply applying the regularization leads to inferior selection performance. This result suggests that the proposed criterion is quite robust to the small sample problem in this classification task. Similar case is observed on the Brodatz data set from Table 2 These results are, more or less, surprising and further analysis leads to the following experiment. By looking into the ORL facial image classification problem, it is found that the total number of training samples is still comparable to the number of features, that is, $5 \times 40=200$ vs. 256 , because there are 40 classes. As defined in the proposed criterion, the radius $R^{2}$ is estimated by using all of the 200 training samples, although the margin $\gamma_{i j}^{2}$ is estimated from the 10 training samples from each pair of classes. Hence, such a training set is not a typical small sample for estimating $R^{2}$. Believing that regularization is necessary in the presence of a small sample problem, this paper 
Table 2. Feature selection performance with regularization (Brodatz data set)

\begin{tabular}{|l||c|c|c|c|c|c|}
\hline \multirow{2}{*}{\multicolumn{1}{|c||}{$\lambda$ value }} & \multicolumn{5}{c|}{ Average test error for different number of selected features (In \%) } \\
\cline { 2 - 7 } & 1 & 2 & 5 & 10 & 15 & 20 \\
\hline \hline 0 & $\mathbf{4 4 . 4 2} \pm \mathbf{6 . 1 1}$ & $\mathbf{2 1 . 5 8} \pm \mathbf{5 . 0 1}$ & $\mathbf{5 . 9 2} \pm \mathbf{4 . 9 1}$ & $\mathbf{3 . 3 3} \pm \mathbf{3 . 2 4}$ & $\mathbf{2 . 0 0} \pm \mathbf{2 . 1 9}$ & $\mathbf{2 . 1 7} \pm \mathbf{1 . 1 2}$ \\
\hline 0.1 & $50.33 \pm 6.70$ & $25.33 \pm 4.32$ & $7.75 \pm 5.31$ & $4.25 \pm 2.95$ & $3.17 \pm 2.32$ & $2.67 \pm 2.07$ \\
\hline 0.25 & $50.42 \pm 6.67$ & $25.00 \pm 4.84$ & $9.42 \pm 3.73$ & $3.58 \pm 1.80$ & $3.25 \pm 2.10$ & $2.67 \pm 2.22$ \\
\hline 0.5 & $50.08 \pm 7.27$ & $23.92 \pm 3.49$ & $9.67 \pm 3.38$ & $4.42 \pm 2.42$ & $2.83 \pm 2.01$ & $2.75 \pm 1.84$ \\
\hline 0.75 & $48.42 \pm 7.76$ & $25.08 \pm 3.98$ & $9.25 \pm 4.09$ & $5.17 \pm 2.99$ & $2.92 \pm 1.72$ & $3.25 \pm 2.10$ \\
\hline 0.9 & $47.58 \pm 8.04$ & $23.58 \pm 3.89$ & $9.42 \pm 4.94$ & $5.58 \pm 2.99$ & $3.08 \pm 2.15$ & $2.50 \pm 1.80$ \\
\hline 0.99 & $47.00 \pm 7.64$ & $24.08 \pm 4.87$ & $8.92 \pm 3.33$ & $5.92 \pm 2.73$ & $3.08 \pm 2.19$ & $2.92 \pm 1.85$ \\
\hline
\end{tabular}

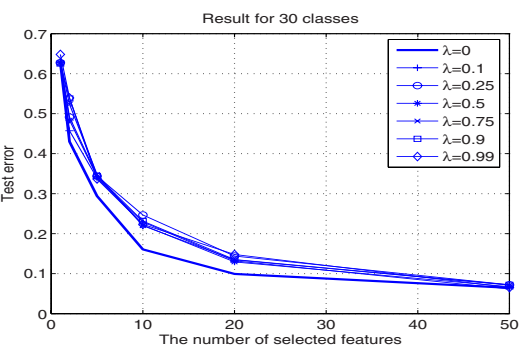

(a) Class number $=30$

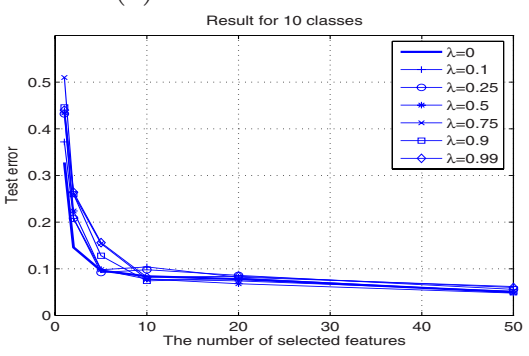

(a) Class number $=10$

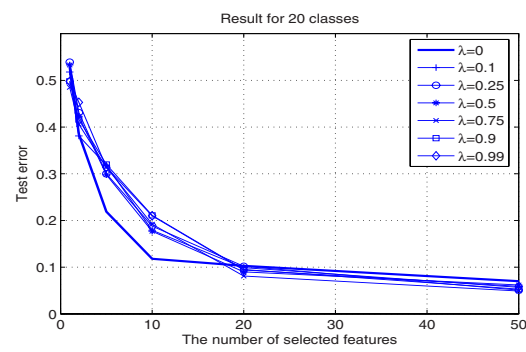

(b) Class number $=20$

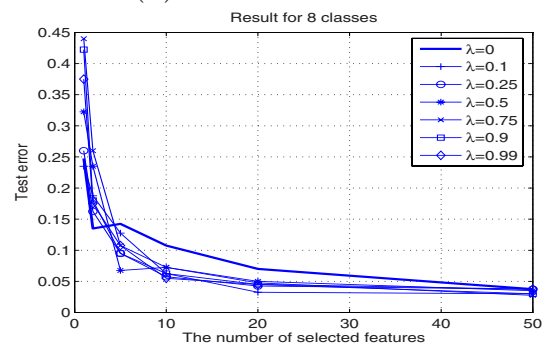

(b) Class number $=8$

Fig. 5. Results for the ORL data set with less number of classes

further conducts the following experiment: maintain 5 training samples in each class and gradually reduce the number of classes to 30, 20, 10 and 8. After that, following the previous procedures, perform feature selection with the proposed criterion for these four new classification problems, respectively. The result is plotted in Figure 5 and it shows what is expected. The selection performance of the criterion without regularization (labelled by $\lambda=0$ and shown as a thick curve) degrades with the decreasing number of classes. When there are only 8 classes (or, equally, $5 \times 8=40$ training samples), the necessity and benefit of regularization can be clearly seen. Summarily, two points can be drawn from Table 12 and Figure 5. (1) thanks to the way of defining $R^{2}$, regularization is not a concern for the proposed criterion when the number of training samples in 
each class is small but the number of classes is large. This case is quite reasonable for multi-class classification; (2) when the total number of training samples is really small, employing the regularized version of the proposed criterion can achieve better feature selection performance.

\section{Conclusion}

A novel feature subset selection approach has been proposed for multi-class SVM based image classification. It handles the selection with hundreds of features, very suitable for image classification where high-dimensional feature vectors are often used. This approach produces overall better performance than the compared selection criteria and shows robustness to the small sample case in multi-class image classification. These results preliminarily verify its effectiveness. More theoretical and experimental study will be conducted in the future work.

\section{References}

1. Kim, K.I., Jung, K., Park, S.H., Kim, H.J.: Support vector machines for texture classification. IEEE Transactions on Pattern Analysis and Machine Intelligence 24(11), 1542-1550 (2002)

2. Chapelle, O., Haffner, P., Vapnik, V.: Support vector machines for histogram-based image classification. IEEE Transactions on Neural Networks 10(5), 1055-1064 (1999)

3. Luo, T., Kramer, K., Goldgof, D., Hall, L., Samson, S., Remsen, A., Hopkins, T.: Recognizing plankton images from the shadow image particle profiling evaluation recorder. IEEE Transactions on Systems, Man and Cybernetics, Part B 34(4), 17531762 (2004)

4. Chapelle, O., Vapnik, V., Bousquet, O., Mukherjee, S.: Choosing multiple parameters for Support Vector Machines. Machine Learning 46(1-3), 131-159 (2002)

5. Wang, L., Chan, K.L.: Learning Kernel Parameters by using Class Separability Measure. In: Presented in the sixth kernel machines workshop, In conjuction with Neural Information Processing Systems (2002)

6. Darcy, Y., Guermeur, Y.: Radius-margin Bound on the Leave-one-out Error of Multi-class SVMs. Technical Report, No. 5780, INRIA (2005)

7. Chang, C.C., Lin, C.J.: LIBSVM: a library for support vector machines (2001), Software available at http://www.csie.ntu.edu.tw/ cjlin/libsvm

8. Schölkopf, B., Smola, A.: Learning with Kernels Support Vector Machines, Regularization, Optimization, and Beyond. MIT Press, Cambridge, MA (2002)

9. Manjunath, B.S., Ma, W.Y.: Texture features for browsing and retrieval of image data. IEEE Transactions on Pattern Analysis and Machine Intelligence 16(8) (1996) 